\title{
Gelebte Traumapädagogik im stationären Setting. Primärprävention von Gewalt und Deeskalation
}

\author{
Andrea Schober
}

Zum Alltag an einer Akutstation für Kinder- und Jugendpsychiatrie gehört die besondere Patientengruppe der Kinder und Jugendlichen, welche traumatisierende Erfahrungen machen mussten. Es handelt sich um Kinder und Jugendliche, die in ihrer frühen Kindheit in den Familien anhaltende Erfahrungen von Gewalt, Verwahrlosung und Vernachlässigung machen mussten. Entwickeln diese Kinder und Jugendlichen dadurch chronische Traumafolgestörungen, kommen sie meist als Patientinnen und Patienten zu einem therapeutischen Aufenthalt, aber auch zu einem Krisenaufenthalt in die Abteilung. Ziel des Aufenthaltes ist zuerst die emotionale und soziale Stabilisierung.

Der alltägliche Umgang und das Miteinander mit den Patientinnen und Patienten in der Gruppe stellt oft das gesamte Team vor große Herausforderungen. Die Grundsätze der traumapädagogischen Arbeit wurden häufig diskutiert.

Ausgestattet mit dem entsprechenden Fachwissen ist es möglich, dass die Kinder und Jugendlichen die adäquate Stabilisierung und Behandlung bekommen, die sie benötigen. Grundlage hierfür ist die Schaffung eines möglichst sicheren Ortes mit verlässlichen und vertrauensvollen Beziehungen. Dabei spielen der Aufbau von Vertrauen und von professionellen Beziehungen sowie die Unterstützung bei der Bewältigung von traumatischen Ereignissen eine wichtige Rolle. Zu Beginn wurden ein kleines multiprofessionelles Team ausgebildet und die Grundsätze der Traumapädagogik als Konzept im Alltag implementiert. Damit die Implementierung an einer kinder- und jugendpsychiatrischen Akutstation gelingen kann, bedarf es unterschiedlicher Voraussetzungen, welche sowohl strukturell als auch organisatorisch und personell auf der Ebene der Mitarbeiterinnen und Mitarbeiter zu denken sind. Allem voran steht jedoch die Etablierung der traumapädagogischen Grundhaltung im gesamten Team.

Die wesentliche Basis der Traumapädagogik stellt die traumapädagogische Grundhaltung dar. Eine wertschätzende und verstehende Haltung bildet das Fundament. Die Patientinnen und Patienten haben in ihrem bisherigen Leben vieles überstanden und sind somit die Expertinnen und Exper- 
ten für sich selbst. Sie schützen sich vor unangenehmen Gefühlen und handeln unbewusst danach. Ihre Verhaltensweisen sind erlernte und dadurch für sie normale Reaktionen, um mit der hohen "Stressbelastung“ überleben zu können. Sie verdienen höchste Wertschätzung, auch wenn dies oftmals schwer anzuerkennen ist: „Du bist, wie du bist, und es ist gut, wie du bist.“

Die Verhaltensweisen der Mädchen und Jungen sind normale Reaktionen auf eine extreme Stressbelastung. Sie haben für ihre Vorannahmen, Reaktionen und Verhaltensweisen einen "guten Grund“. Sie haben in ihrem Leben bislang viel überstanden und geleistet. Wir unterstützen sie bei der Akzeptanz ihrer seelischen Wunden, Beeinträchtigungen und Schwierigkeiten. Wir stellen unser Fachwissen zur Verfügung, doch sie sind selbst die Expertinnen und Experten für ihr Leben, für das Bestehen in schwierigen Lebenslagen (Weiß 2013).

\section{Definition Psychotraumatologie}

Analog und in Abgrenzung zur somatischen Traumatologie (von griech. Trauma $=$ Verletzung) steht der Begriff der Psychotraumatologie. Dieser befasst sich mit der Entstehung, der Erfassung, dem Verlauf und der Behandlung von seelischen Verletzungen, die als Folge extrem belastender und/oder lebensbedrohlicher Ereignisse auftreten (Landolt 2004). Leonore Terr (1991) unterscheidet Typ-1- und Typ-2-Traumata. Unter Typ 1 versteht man akute, unvorhergesehene und einmalige Ereignisse, wie zum Beispiel einen Unfall oder eine Geiselnahme. Typ-2-Traumata treten wiederholt oder anhaltend auf und sind teilweise vorhersehbar (Terr 1991).

Die Kenntnisse der verschiedenen Traumata und der unterschiedlichen Wirkfaktoren bilden die Grundlage für eine angemessene Hilfe (Weiß 2008). Anette Engfer definiert folgende gesicherte Risikofaktoren:

- emotionale, körperliche oder sexuelle Misshandlung,

- elterlicher Alkohol- und Drogenmissbrauch,

- ärmliche Verhältnisse, elterlicher Verlust der Arbeit,

- Trennung/Scheidung/Wiederverheiratung/väterliche Abwesenheit,

- mütterliche Berufstätigkeit im ersten Lebensjahr,

- körperliche und/oder psychische Erkrankungen der Eltern,

- chronische familiäre Disharmonie,

- Kriminalität und Dissozialität eines Elternteiles,

- Umzüge, Schulwechsel, 
- ernste Erkrankungen in der Kindheit und

- körperliche Gewalt in der Familie. (Engfer 2000, 14)

Als weitere Risikofaktoren gelten emotionale und körperliche Vernachlässigung, anhaltende Abweisung, häusliche Gewalt, ungebührliche elterliche Machtausübung, wie zum Beispiel das Münchhausen-Stellvertreter-Syndrom, Unfälle, Krankenhausaufenthalte, (gewaltsamer) Tod eines Familienangehörigen, Obdachlosigkeit, Flucht, Krieg, Naturkatastrophen sowie körperliche und geistige Behinderungen (Weiß 2008, 21).

Stationäre Behandlung von traumatisierten Kindern und Jugendlichen bedeutet, zwischenmenschliche, räumliche und institutionelle Perspektiven zu erkennen und diese umzusetzen, damit eine fachliche Kontinuität und Stabilität in personeller und in struktureller Hinsicht gewährleistet werden kann. Es entstehen institutionelle Anforderungen an die Leitungsebene. Das Organisationsmanagement innerhalb der Abteilung muss ständig evaluiert werden. Komplexe Anforderungen an jeden einzelnen Mitarbeiter und jede einzelne Mitarbeiterin sowie an das gesamte multiprofessionelle Team müssen berücksichtigt werden.

\section{Anforderungen an die Institution und die Führungskräfte}

Die innere Grundhaltung im Sinne von Überzeugungen und Wertvorstellungen von Menschen bildet sich über einen langen Zeitraum heraus und wird über frühe Erfahrungen im sozialen Umfeld erworben und im Erwachsenenalter stabil gehalten. Diese kann nur sehr langfristig verändert werden. Gerade unter Belastung und Stress halten Menschen an ihren ureigenen Wertvorstellungen fest.

Deshalb braucht es nicht nur Qualifikationen, sondern auch eine wertorientierte Organisationseinheit, dass die Mitarbeiterinnen und Mitarbeiter im Alltag Orientierung und Führung erfahren (Schirmer 2013, 246).

- Leitbild: Die gemeinsame Erstellung eines Leitbildes mit dazugehörigen Leitsätzen ist unumgänglich. Definiert werden müssen darin Atmosphäre, pädagogische Haltung, Pädagogik und Individualität, Würde, Wertschätzung, Vorbild, Lebensfreude, Spiritualität, Überzeugungen sowie Art und Weise des Umgangs miteinander - nach innen und außen.

- Qualitäts- und Risikomanagement: Definition von Risiken, Prozessabläufe, Krisenmanagement. Richtlinien und Serviceunterlagen müssen für jeden Mitarbeiter und jede Mitarbeiterin klar sein. Konzepte für al- 
le Teilbereiche müssen aufliegen und die Mitarbeiterinnen und Mitarbeiter müssen danach ihre Handlungsabläufe richten.

- Personalmanagement: Das Team ist multiprofessionell und besteht aus Ärztinnen und Ärzten, Psychologinnen und Psychologen, Gesundheitsund Krankenpflegepersonal, Psychotherapeutinnen und -therapeuten, Sozialpädagoginnen und -pädagogen, Sozialarbeiterinnen und -arbeitern sowie Lehrerinnen und Lehrern. Die Mitarbeiterinnen und Mitarbeiter müssen über eine hohe Reflexionsfähigkeit, Belastbarkeit, Beziehungs- und Konfliktfähigkeit, Humor sowie emotionale Stabilität verfügen. Besonderes Augenmerk muss man auf ausreichende Personalressourcen richten.

- Bauliche und räumliche Gegebenheiten sollten so gestaltet sein, dass folgende Rahmenbedingungen erfüllt werden können:

- Sicherung der Grundbedürfnisse und Wahrung der Intimsphäre

- Zimmer (Einzelzimmer) als Rückzugsort - können durch die Patientinnen und Patienten selbst gestaltet werden

- Wohlfühlatmosphäre in den Gruppenräumen

- Schutz vor dem Wiedererleben traumatischer Erfahrungen - siehe „sicherer Ort"

- Klarheit, Übersichtlichkeit, Orientierung

- Organisations- und Zeitmanagement in der Tages- und Wochenstruktur des jahreszeitlichen Kreislaufes müssen durch die Führungskräfte gesichert werden:

- Sicherung der 24-Stunden-Betreuung im stationären therapeutischen Bereich und im Akutsetting (auch im Unterbringungsbereich)

- Bezugs-/Beziehungspflege/Pädagogik

- professioneller Beziehungsaufbau/Einzelbetreuungen

- Alltagsgestaltung

- gruppendynamische Herausforderungen - alltägliches Zusammenleben der Gesamtgruppe

- Koordination des multiprofessionellen Behandlungsteams: Die Kompetenzen sind berufsgruppenspezifisch, die "gemeinsame Sprache“ bildet die Basis. Menschliche Faktoren spielen dabei eine tragende Rolle, aber auch grundsätzliche Einstellungen und das zwischenmenschliche Klima. 


\section{Anforderungen an die Mitarbeiterinnen und Mitarbeiter}

In erster Linie geht es um Vertrauen und einen guten Austausch im Behandlungsprozess! Die Mitarbeiterinnen und Mitarbeiter müssen so gestärkt und/oder befähigt werden, dass sie ihre Aufgaben selbstverantwortlich lösen können. Dazu zählt der Erwerb von drei Grundkompetenzen für professionelles Handeln:

- Sachkompetenz: Diese erzielen die Mitarbeiterinnen und Mitarbeiter durch die Aneignung von spezifischem Fachwissen.

- Selbstreflexion: Diese wird allgemein als eine der wichtigsten Kompetenzen in der sozialen Arbeit bezeichnet. Ihr Ziel ist es, die eigenen Verarbeitungsmuster zu erkennen und Belastungen $\mathrm{zu}$ reduzieren. Kindheitsgeschichten, Bindungsmuster, Geschlechterrolle, Sexualität und Motive des eigenen Handelns sollten Themen sein.

- Selbstfürsorge: Sie ist ein notwendiger Bestandteil der beruflichen Identität zum Erhalt der psychischen Gesundheit oder zur Verhinderung von Burnout. (Weiß 2008, 196 ff.)

\subsection{Traumapädagogische Haltung - Haltungselement auf Mitarbeiterebene}

Wir müssen unsere Patientinnen und Patienten mit ihrer Geschichte annehmen. Die Anpassungsbemühungen müssen als Überlebensstrategie verstanden werden (Aggression als Ressource). Interventionen, welche die Grundgefühle und Grundannahmen verstärken, müssen vermieden werden. Respekt vor der Lebensleistung unserer Patientinnen und Patienten ist oberstes Gebot (Weiß 2008).

\section{Schutzfaktoren/Skills der Mitarbeiterinnen und Mitarbeiter}

In der Arbeit mit traumatisierten Patientinnen und Patienten ist es notwendig, die Mitarbeiterinnen und Mitarbeiter vor den spezifischen Belastungen durch kompensatorische Schutzfaktoren zu schützen (Weiß 2008, 206). 


\subsection{Fachlichkeit der Mitarbeiterinnen und Mitarbeiter-Fortbildungen}

Ein umfassendes Wissen über allgemeine Störungsbilder im Kindes- und Jugendalter, insbesondere im Bereich der Psychotraumatologie, muss vorhanden sein. Dazu sind regelmäßige fachspezifische Fortbildungen im Bereich Traumapädagogik, Gesprächsführung/ Kommunikation, Deeskalations- und Sicherheitsmanagement erforderlich. Die Sicherung des Fortbildungstransfers muss gewährleistet sein, etwa in Form von abteilungsinternen Fortbildungen, gemeinsamen Fallbesprechungen, Intervisionen oder Supervisionen. Durch die regelmäßigen Fortbildungen sollen die Mitarbeiterinnen und Mitarbeiter die Fähigkeit bekommen, die emotionalen Grundbedürfnisse der Patientinnen und Patienten rechtzeitig zu erkennen, darauf einzugehen und danach zu handeln.

\subsection{Supervision}

Die Mitarbeiterinnen und Mitarbeiter müssen die Möglichkeit bekommen, regelmäßige Supervisionen zu besuchen. Die Supervidierung der Arbeit ist wichtig, um zum Beispiel stellvertretende Traumatisierungen zu thematisieren. Des Weiteren können „Tabu-Themen“ wie Gegenübertragungsgefühle, Belastungen oder Verwicklungen in das eigene Schicksal angesprochen werden, und das im geschützten Rahmen (Weiß 2008, 208).

\section{Ausgewählte Aspekte der Traumapädagogik}

\subsection{Der sichere Ort}

Die Traumapädagogik beschreibt als Antwort auf das Erleben in den traumatisierenden Situationen und die daraus resultierenden Folgen wie Körperreaktionen, Emotionsregulation und Verhaltensstrategien die Notwendigkeit zur Bereitstellung eines „sicheren Ortes“, an dem die betroffenen Patientinnen und Patienten neue korrigierende Erfahrungen mit sich und ihrer Umwelt machen können, um sich neue Verhaltensstrategien erarbeiten zu können (Lang 2013, 136). Dieser sichere Ort wird in der Triade Struktur - Mitarbeiter/-innen - Patientinnen/Patienten beschrieben, wobei alle drei Aspekte in ihrer Bedeutung gleich zu gewichten sind (Lang et al. 2009). 


\begin{tabular}{|c|c|c|c|c|}
\hline & $\begin{array}{l}\text { Balance der } \\
\text { Sinnes- } \\
\text { wahr- } \\
\text { nehmung }\end{array}$ & $\begin{array}{l}\text { Emotions- } \\
\text { regulation }\end{array}$ & $\begin{array}{l}\text { Ressourcen- } \\
\text { stärkung }\end{array}$ & $\begin{array}{l}\text { Selbst- } \\
\text { wirk- } \\
\text { samkeit }\end{array}$ \\
\hline STRUKTUR & $\begin{array}{l}\text { Farben / } \\
\text { Materialien / } \\
\text { heile } \\
\text { Umgebung }\end{array}$ & Gefühlsraum & $\begin{array}{l}\text { Einzel= } \\
\text { betreuung }\end{array}$ & $\begin{array}{l}\text { Hoheit über } \\
\text { den eigenen } \\
\text { Bereich }\end{array}$ \\
\hline $\begin{array}{l}\text { MIT= } \\
\text { ARBEITER }\end{array}$ & $\begin{array}{l}\text { Imaginations,- } \\
\text { Achtsam= } \\
\text { keitsübungen } \\
\text { Freundlichkeit }\end{array}$ & $\begin{array}{l}\text { Emotionales } \\
\text { Versorgungs= } \\
\text { team }\end{array}$ & $\begin{array}{l}\text { Resilienz= } \\
\text { orientierte } \\
\text { Weiter= } \\
\text { bildung }\end{array}$ & $\begin{array}{l}\text { Selbst= } \\
\text { reflexion } \\
\text { „hilflose } \\
\text { Helfer“ }\end{array}$ \\
\hline $\begin{array}{l}\text { KINDER/ } \\
\text { JUGEND- } \\
\text { LICHE }\end{array}$ & $\begin{array}{l}\text { Massagen / Öle } \\
\text { Aufmerksam } \\
\text { machen }\end{array}$ & $\begin{array}{l}\text { Gefühle } \\
\text { kennenlernen, } \\
\text { unter= } \\
\text { scheiden }\end{array}$ & $\begin{array}{l}\text { Aktivitäten, die } \\
\text { stark machen }\end{array}$ & Notfallkoffer \\
\hline
\end{tabular}

Abb. 1: Der sichere Ort (vgl. Lang et al. 2009)

An diesem sicheren Ort können die Patientinnen und Patienten positive Beziehungserfahrungen machen und emotional nachreifen. Entwicklungsschritte können nachgeholt und Selbstwirksamkeitserfahrungen gemacht werden. Wenn das gelingt, können sich die Kinder und Jugendlichen den eigenen Entwicklungsaufgaben und den Anforderungen der Gesellschaft stellen und diese auch bewältigen.

\subsection{Selbstbemächtigung}

Darunter versteht man, dass sich das Kind bzw. der oder die Jugendliche nicht länger als Objekt, sondern als handlungswirksames Subjekt erlebt (Weiß 2013).

\subsubsection{Das Selbstverstehen - das „dreigliedrige Gehirn“}

Zum Selbstverstehen zählt, dass die Kinder und Jugendlichen erkennen und wissen, dass Gewalt gegen Kinder häufig vorkommt und dass sie nicht schuld daran sind, sondern die Verantwortung bei den Erwachsenen liegt. Sie müssen verstehen lernen, dass die früheren Erfahrungen noch immer wirken und das Heute beeinflussen. Sie müssen wissen, wie der Körper 
und der Kopf reagieren. Sie müssen gesellschaftliche Strukturen und deren Wirksamkeit erkennen (zum Beispiel Arm und Reich). Die Förderung dieses Wissens und des Selbstverstehens kann über das „dreigliedrige Gehirn“ veranschaulicht werden:

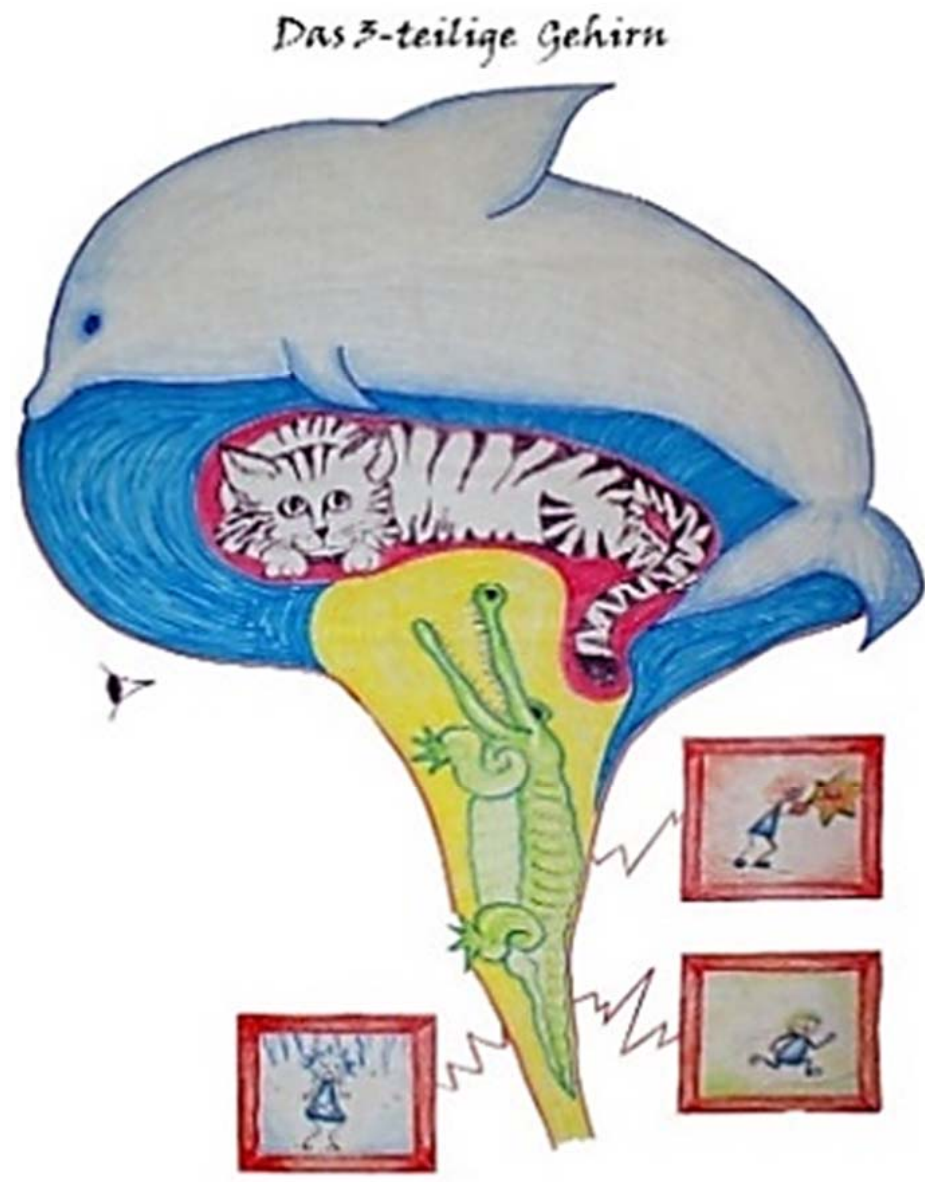

Abb. 2: Das dreigliedrige Gehirn (Traumapädagogisches Team KJP Graz, 2016)

Die Katze beschäftigt sich mit deinen alltäglichen Gefühlen.

Der Delfin denkt nach.

Das Krokodil ist der Autopilot für deinen Körper. 
In sehr belastenden Situationen ist die Katze mit großen Gefühlen überfordert. Das Krokodil ist aktiv und löst Notfallprogramme aus (Kämpfen, Flüchten, Erstarren). Der Delfin kann nicht mehr denken. Es ist möglich, das Krokodil zu verstehen, die Katze zu beruhigen und einen Zugang zum Delfin zu finden. Somit kriegt man wieder Kontrolle über das Verhalten (Traumapädagogisches Team KJP Graz, 2016).

\subsubsection{Die Selbstakzeptanz - das hilfreiche Wort "weil“ und der „gute Grund“}

Die Akzeptanz von eigenen Schwierigkeiten, Wunden und Beeinträchtigungen ist Bestandteil der Traumaheilung. Die Unterstützung dieser Akzeptanz ist Bestandteil der traumapädagogischen Grundhaltung. Die Kinder und Jugendlichen haben einen guten Grund, so zu handeln, wie sie handeln. Und nun muss man den ganz eigenen Grund herausfinden. Dazu eignet sich das Wörtchen „weil“ ganz gut. Das Wort „weil“ lädt zum Antworten ein und transportiert eine wertschätzende Haltung: „Du tust das, weil ...?“ Die Annahme des guten Grundes lädt ein, über sich nachzudenken. Das Erkennen des guten Grundes ist Voraussetzung, um neue, weniger selbst- und fremdschädigende Verhaltensweisen entwickeln zu können (Weiß 2013, 149 ff.).

\subsubsection{Die Selbstwahrnehmung - „Ich bin Kurt“}

Selbstwahrnehmung ist die Wahrnehmung des Selbst und der eigenen Person. Die Sensibilisierung für die Körperempfindungen und Gefühle muss wieder erlernt werden. Traumatisierte Kinder und Jugendliche haben gelernt, ihre Gefühle abzuspalten. Abgespaltene Gefühle wie Wut oder Angst müssen wieder bewusst gemacht und integriert werden. Dazu eignen sich verschiedene Methoden (Weiß 2008, 110). An der KJP Graz gibt es dazu „Kurt“. Bei Kurt kann das Kind bzw. der oder die Jugendliche seine Ängste und Gefühle zum Ausdruck bringen und sie auch zeichnen. Wo im Körper nehme ich Stress wahr? Wo kribbelt es gerade? Wo wird mir heiß? Es entsteht eine persönliche Landkarte von Körperempfindungen bei Angst, Traurigkeit, Wut etc. - wie fühlt sich das in meinem Körper an? 


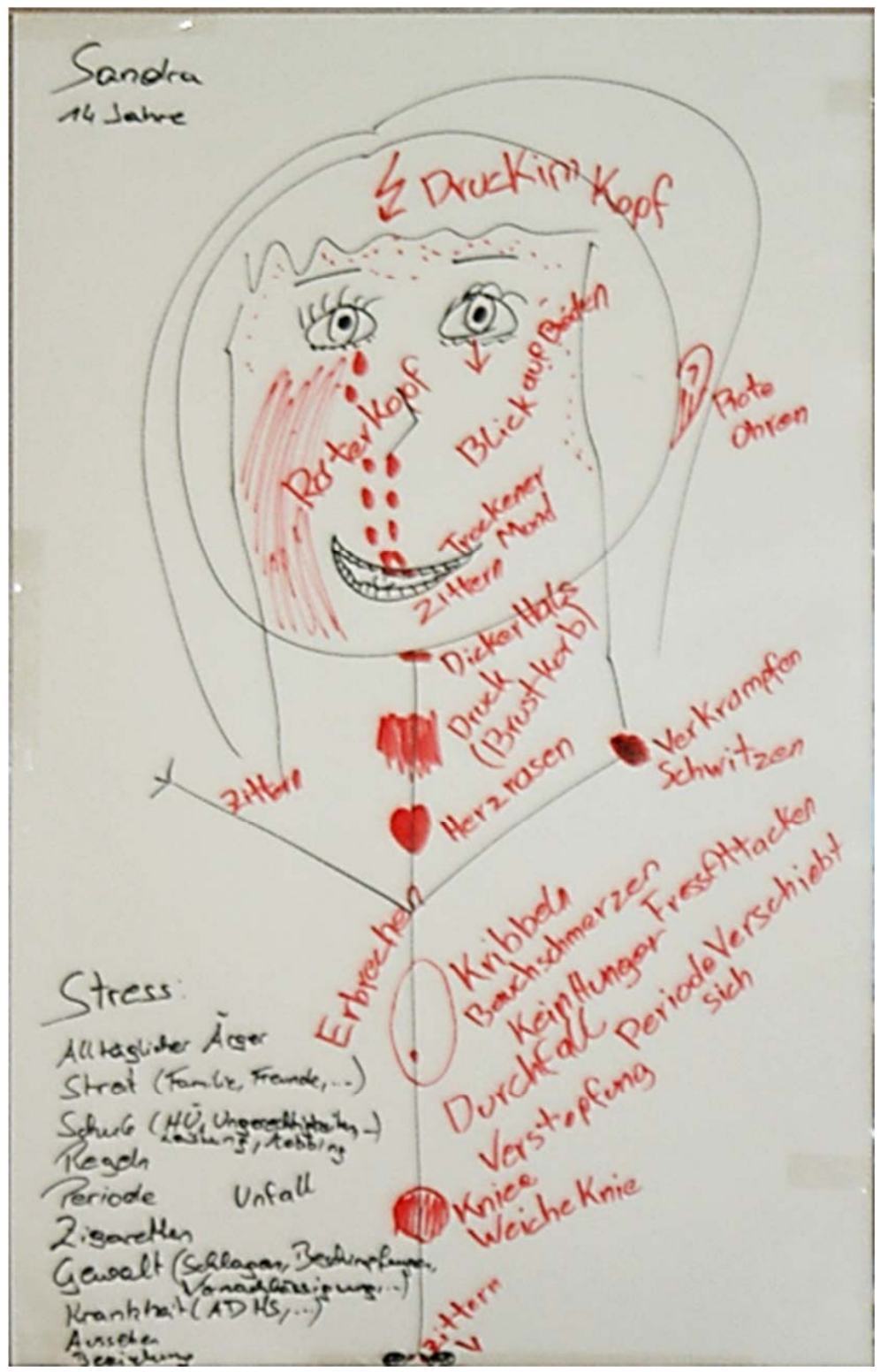

Abb. 3: Persönliche Landkarte von Körperempfindungen (Traumapädagogisches Team KJP Graz, 2016) 


\subsubsection{Die Selbstregulation - der innere sichere Ort}

Traumatisierte Kinder und Jugendliche brauchen einen „inneren sicheren Ort“, um zu lernen, wie sie sich selbst zur Ruhe bringen, an sich glauben und sich selbst regulieren können. Selbstregulation umfasst den mentalen Umgang mit Gefühlen, Stimmungen und körperlichen Manifestationen (Weiß 2008, 113). Der innere sichere Ort definiert sämtliche notwendige Fähigkeiten zur Emotionsregulation, zum Spannungsabbau und zur Selbstberuhigung.

Bei der Förderung der Selbstregulation geht es um die Stabilisierung von Stressoren und um das Wahrnehmen, Erkennen und Versorgen von sekundären Traumasymptomen (Levine/Kline 2011):

- gezielte Förderung der Körper- und Sinneswahrnehmung,

- sprachliche Benennung von Empfindungen,

- Unterscheidung von angenehmer und unangenehmer Nähe,

- erlernen, Trigger zu benennen.

In der Praxis kommen folgende Techniken zur Anwendung: Atemübungen, Selbstmassagen, Körperübungen, Gedankenstopptechniken, starke Sinnesreize, Entspannendes - alles, was „guttut“.

Der „persönliche“ Notfallkoffer oder Skills kommen dabei zum Einsatz. Diese sind sehr individuell und werden in stressfreien Zeiten geübt und trainiert. 

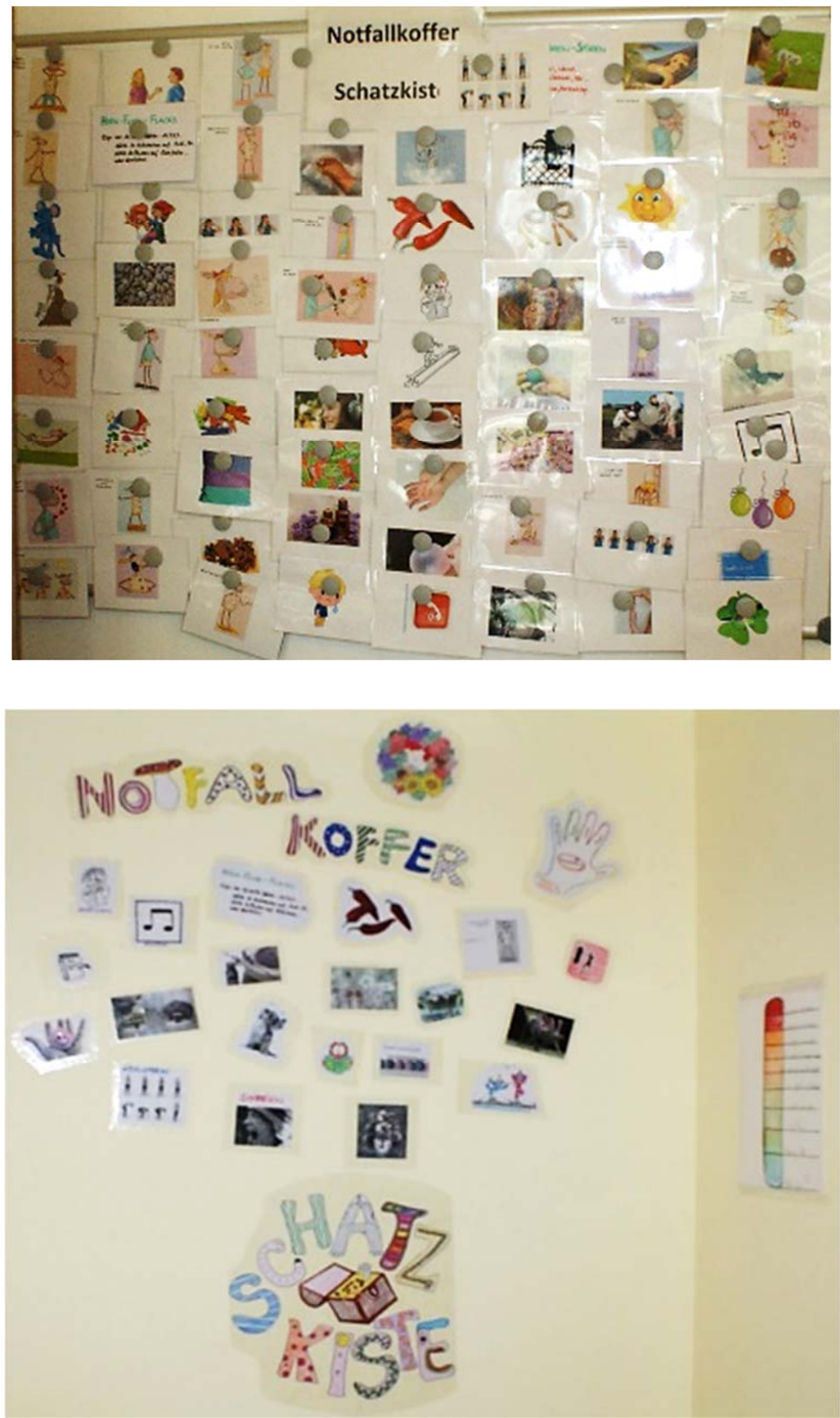

Abb. 4 und 5: Notfallkoffer (Traumapädagogisches Team KJP Graz, 2016) 


\subsection{Transparenz}

Transparenz verfolgt das Anliegen, Orte der Unberechenbarkeit zu Orten der Berechenbarkeit werden zu lassen. Für die Patientinnen und Patienten wird der Alltag durch regelmäßige Abläufe und die zeitliche Verfügbarkeit der Bezugspflege klar und berechenbar. „Ich habe jederzeit das Recht auf Klarheit!"

Die Berechenbarkeit, die Klarheit, die Vorhersehbarkeit sowie die Verstehund Begründbarkeit schaffen Sicherheit (Lang et al. 2013, 118 ff.):

- transparente Abläufe im Alltag

- Erarbeitung von Wochenzielen

- Tagesstrukturplan - Was passiert heute?

- öffentlicher Dienstplan - Wer ist heute für mich da?

- Bezugspflege/Pädagogik - Wer ist er/sie? Was passiert, wenn er/sie frei hat? Wer hat heute Nachtdienst?

- transparente Kommunikation

- Welche Regeln gelten hier? - Stationsregeln - Warum sind die so?

- Was passiert bei Grenzüberschreitungen?
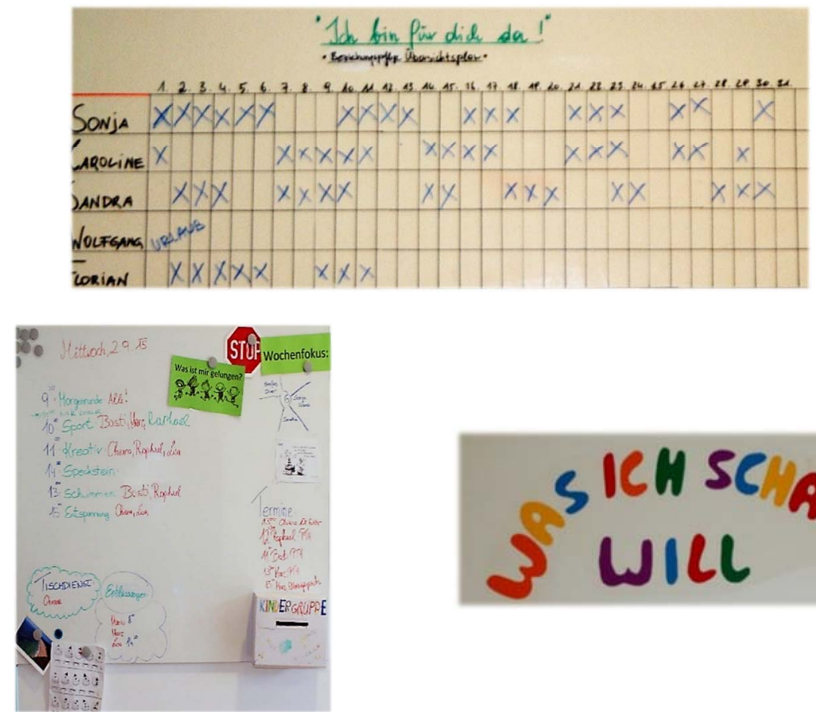

Abb. 6, 7 und 8: Transparenz im Alltag (Traumapädagogisches Team KJP Graz, 2016) 
Ein Einblick in die transparenten "goldenen Stationsregeln“ an der KJP Graz:

- Ich halte meinen Tagesablauf ein!

- Ich entferne mich nicht, ohne zu fragen, von der Station oder Schule!

- Ich höre auf die Anweisungen des Personals bezüglich Aktivitäten, Ausflüge, Schule und Station!

- Mädchenzimmer sind für Buben tabu! Bubenzimmer sind für Mädchen tabu!

- Schlagen, Treten und Spucken sind verboten!

- Rauchen ist laut Jugendschutzgesetz nicht erlaubt!

- Alkohol, Drogen und aufputschende Getränke sind verboten!

Bei Nichteinhaltung gelten transparente Regeln:

- Benennen der Regelverletzung

- Ankündigen der Konsequenz

- Chance geben

- bei Änderung des Verhaltens loben

- keine Änderung: Durchführung der Konsequenz

- aus der Situation nehmen oder „Time-out“ - Diese Entscheidung ist besonders in Gruppen wichtig abzuwägen.

- Wenn ein Kind bzw. ein Jugendlicher oder eine Jugendliche aus der Gruppe gehen muss, darf diese Zeit nicht zur „Freizeit“ werden. $\rightarrow$ eventuell Verstärkung (Traumapädagogisches Team KJP Graz, 2016)

Allgemeine pädagogische Haltung und Vorgehen:

- Konsequenzen müssen

- durchführbar,

- unmittelbar (bei Gefahr immer) und

- mit dem Problemverhalten verbunden sein.

- Nach Möglichkeit sollen sie von jener Person durchgeführt werden, welche sie ausspricht (Traumapädagogisches Team KJP Graz, 2018).

\subsection{Milieutherapeutische Konzepte}

Das „therapeutische Milieu“ findet im Alltag statt. Bindungs- und Beziehungsarbeit stellt die Kernkompetenz dar, Strukturgebung bildet das fundamentale Gegenüber. Die Milieugestaltung spannt einen Bogen von zwi- 
schenmenschlichen zu räumlichen und institutionellen Perspektiven. In einem klar strukturierten Alltag können die Patientinnen und Patienten modellhaft erlernen, dass mit Unterstützung durch das Team und die Gruppe ein weiterer Schritt im Leben zu bewältigen ist. Im Zentrum des Alltags der Patientinnen und Patienten stehen Wahrnehmungs-, Interaktions- und Kommunikationsprozesse (Gahleitner 2012, 9 ff.).

Bei einer Betroffenenbefragung stellten sich folgende Aspekte des therapeutischen Milieus als wirksam heraus:

- das unmittelbar und im Alltag stattfindende Betreuungsangebot als Basis des therapeutischen Milieus („Sie waren halt für einen da. “),

- die professionelle Bindungs- und Beziehungsarbeit als Kernkompetenz,

- die angemessene Strukturgebung („Da schaut einer auf mich haargenau, da kriegt man dann Vertrauen. "),

- die Therapie - eingebettet in den Gesamtkontext (Gahleitner 2014).

Zusammenfassend treffen folgende Kernaussagen auf das therapeutische Milieu zu:

- viel Verständnis für die Nöte und Ängste der Patientinnen und Patienten und/oder der Mitarbeiterinnen und Mitarbeiter,

- kleine, gut strukturierte, homogene, mit hohem Personalschlüssel ausgestattete Gruppen,

- ein eng geknüpftes Netz aus Bindungsbezügen, geprägt durch Fachwissen und Sozialisationsstrukturen,

- hohe Kompetenz im medizinisch-pflegerisch-pädagogischen Handeln und therapeutischen Verstehen.

Das Behandlungswerkzeug ist das Team, nicht ein OP-Saal.

\subsection{Traumatische Übertragung und Gegenreaktion}

Kinder und Jugendliche, die in ihrer Lebensgeschichte durch ihre Bindungspersonen traumatisiert wurden, übertragen ihre traumatischen Erfahrungen und Erlebnisse auf ihre neuen Interaktionspartner. In der Regel sind es desorganisierte und hochunsichere Bindungserfahrungen. Ihre Lebensgeschichte ist geprägt durch eine drohende existentielle Vernichtung. Ihre früheren Erfahrungen, durch Bezugspersonen verletzt worden zu sein, steigert die Angst vor neuen Verletzungen (Lang 2013, 194). Judith Hermann schreibt: 
„Im Augenblick des Traumas ist das Opfer ganz und gar hilflos. Unfähig sich zu verteidigen, schreit es nach Hilfe, aber niemand kommt. Es fühlt sich völlig verlassen. Die Erinnerung an diese Erfahrung prägt alle späteren Beziehungen.“ (Hermann 2010, 188).

Die emotionalen Reaktionen der Kinder und Jugendlichen verlagern sich in der Übertragungsbeziehung vom Täter auf die Mitarbeiterinnen und Mitarbeiter in der Abteilung, durch die sie nun begleitet werden (Lang 2013, 193). In der traumatischen Übertragung fühlt sich das Kind bzw. der oder die Jugendliche bedroht, hilflos, ohnmächtig und wütend. Sie erzählen von diesen Erfahrungen und zeigen Flucht-, Kampf- und Erstarrungsstrategien. So fühlen sich dann die Mitarbeiterinnen und Mitarbeiter hilflos, beschämt, wütend ...

Durch die Übertragungsbeziehung werden die Mitarbeiterinnen und Mitarbeiter oft von ihren eigenen Gefühlen überwältigt. Die aufkommenden Anpassungsstrategien als Reaktion auf die Verhaltensweisen der Kinder und Jugendlichen sind Gegenreaktionen. In der traumatischen Gegenreaktion fühlen sich die Mitarbeiterinnen und Mitarbeiter bedroht und hilflos. Sie zeigen unbeabsichtigt Verhaltensmuster der Täter-Opfer-Dynamik. Das Kind bzw. der oder die Jugendliche fühlt sich bedroht, hilflos usw. (Lang 2013, 196). Nun gilt es, dass sich die Mitarbeiterinnen und Mitarbeiter „versorgen“. Sie sollten bei sich bleiben, sich versorgen und stabilisieren. So kann die Versorgung der Patientinnen und Patienten gelingen. Die Bedürfnisse der Patientinnen und Patienten werden erkannt, der Kontakt mit dem Kind bzw. dem oder der Jugendlichen bleibt aufrecht und es erfolgt die versorgende, stabilisierende Intervention für die Patientinnen und Patienten (Lang 2013, 208).

\section{Deeskalationsprävention durch Traumapädagogik}

Wie wir in der Abteilung erkennen durften, wirkt eine gelebte Traumapädagogik als Deeskalationsmethode im Alltag sehr gut. Werden die traumapädagogischen Standards eingehalten, kann man dadurch Affektdurchbrüche der Kinder und Jugendlichen in Grenzen halten. Die Grundhaltung im Team muss jedoch von einem einheitlichen Verständnis von Regeln und Grenzen geprägt sein. Trotzdem muss die Individualität der Patientinnen und Patienten sowie der unterschiedlichen Probleme und Krankheitsbilder berücksichtigt werden - jeder bekommt das, was er braucht. Die ausgewählten Aspekte der Traumapädagogik müssen gelebt werden. In einer Akutabteilung gilt es jedoch zu bedenken, dass nicht alle Kinder 
und Jugendlichen auf freiwilliger Basis in die Abteilung kommen. Es sollte alles versucht werden, den Kindern und Jugendlichen den freiwilligen Weg in die Abteilung zu ermöglichen. Ist dies aber nicht möglich, bleibt oftmals die Zwangseinweisung als einzige Alternative, um eine akute Selbst- oder Fremdgefährdung auszuschließen. Gegen den Willen und womöglich von der Polizei in ein stationäres Setting gebracht zu werden, stellt ein einschneidendes Erlebnis dar. Vor allem in diesen Situationen sind Deeskalations- und Sicherheitsmaßnahmen sowie eine deeskalierende Kommunikation eine absolute Notwendigkeit.

Was ist bei der nonverbalen Kommunikation zu berücksichtigen? Der Einsatz der Augen als Kommunikationsmittel - die Auswirkung von Augenkontakt sollte nicht unterschätzt werden. Vor allem in Phasen hoher Erregung kann direkter Blickkontakt bedrohlich wirken. Körperliche Nähe und Distanz müssen flexibel eingesetzt werden. Bei Berührungen und Körperkontakt muss die Geschichte des Kindes im Auge behalten werden, um Retraumatisierungen zu vermeiden. Ein wichtiges Element ist die Körpersprache. Die Betreuungsperson nimmt die Sitzhaltung des Gegenübers ein und geht immer mehr in eine entspannte Körperhaltung über. Gestik und Mimik können unterstützend eingesetzt werden. Es gilt jedoch immer, sowohl die verbale als auch die nonverbale Kommunikation aufrechtzuerhalten. Dies kann durch folgende Punkte gelingen: Wertschätzung signalisieren, dosiert reden, das Schweigen konstruktiv nutzen, Blockaden erkennen und nutzen, einfühlsame Feedbacks geben, „Ich-Botschaften“ ohne Vorwürfe zu machen, auf Gefühle und Erlebnisinhalte eingehen. Erst bei Entspannung kann ein reflektierendes 4-Augen-Gespräch geführt werden, in dem auch alternative Handlungsweisen aufgezeigt werden können (Traumapädagogisches Team KJP Graz, 2016).

\subsection{Grundhaltung bei Affektdurchbruch an der KJP Graz}

Keine Diskussion soll im Affekt stattfinden! Den Patientinnen und Patienten muss eine Rückzugsmöglichkeit in Form eines Time-out angeboten werden. Das Problemverhalten und die daraus resultierenden Konsequenzen werden danach in Ruhe besprochen. Ein Affektdurchbruch schützt nicht vor Konsequenzen! 


\subsubsection{Was ist danach wichtig?}

Ein strukturiertes Krisenmanagement - CISM (Stressbewältigung nach belastenden Ereignissen) - muss zum Einsatz kommen. Eine multiprofessionelle Fallbesprechung im interdisziplinären Austausch sollte einem Affektdurchbruch folgen. Sind Mitarbeiterinnen und Mitarbeiter schwer belastet und das interne Krisenmanagement nicht ausreichend, muss das Angebot eines externen Coachings und/oder einer Supervision folgen.

\subsubsection{Exkurs: Stressbewältigung nach belastenden Ereignissen}

Es handelt sich dabei um Standardisierungen von Interventionsabläufen nach belastenden Ereignissen. Zum Einsatz kommt eine selbstständige Durchführung der Interventionen von ausgebildeten Peers innerhalb der Abteilungen. Die Kenntnis des verbindlichen Ablaufplanes für Stressbewältigung nach belastenden Ereignissen, psychologische Nachbearbeitungen und Nachsorgemaßnahmen ist für jeden Mitarbeiter verpflichtend.

Des Weiteren müssen Kinder- und Jugendrechte in der Kinderschutzgruppe offensiv behandelt werden. Bei Grenzverletzungen durch das betreuende Personal muss ein klares Verfahren zum Einsatz kommen. Das Beschwerdemanagement, das Leben einer Fehlerkultur und der Leitfaden zum Umgang mit Gewalt sind ebenso Maßstab wie der traumapädagogische Standard (Multiprofessionelles Team KJP Graz, 2018).

\section{Schlussfolgerung}

Wir in der Abteilung für Kinder- und Jugendpsychiatrie und Psychotherapie müssen dafür Sorge tragen, dass der professionelle Umgang mit traumatisierten Kindern und Jugendlichen sowohl diesen nützt als auch uns selbst nicht schadet. Mit Grundkompetenzen wie Sachkompetenz, Selbstreflexion und Selbstfürsorge kann die Professionalität erhöht werden. Spezifische Weiterbildungen für das gesamte multiprofessionelle Team sind absolut erforderlich. Eine individualisierte und an den einzelnen Bedürfnissen der Patientinnen und Patienten angepasste gemeinsame Haltung schafft Entwicklungspotential.

Grundwerte wie Transparenz, Partizipation, Wertschätzung, die Annahme des guten Grundes sowie Freude und Spaß müssen vor Ort authentisch gelebt werden. So kann Traumapädagogik gelingen! 


\section{Literatur}

Baier, Martin: Herausforderung Alltag. Praxishandbuch für die pädagogische Arbeit mit psychisch gestörten Jugendlichen, Göttingen: Vandenhoeck \& Ruprecht ${ }^{2} 2010$.

Bundesarbeitsgemeinschaft Traumapädagogik (BAG TP): Standards für traumapädagogische Konzepte in der stationären Kinder- und Jugendhilfe. Ein Positionspapier der BAG Traumapädagogik, Gnarrenburg: BAG 2011.

Engfer, Anette: Formen der Misshandlung von Kindern - Definitionen, Häufigkeiten, Erklärungsansätze, in: Egle, Ulrich Tiber/Hoffmann, Sven Olaf/Joraschky, Peter (Hg.): Sexueller Missbrauch, Misshandlung, Vernachlässigung. 2., vollst. aktual. u. erw. Aufl., Stuttgart/New York: Schattauer 2000, 3-19.

Gahleitner, Silke Brigitta/Frank, Christina/Leitner, Anton (Hg.): Ein Trauma ist mehr als ein Trauma. Biopsychosoziale Traumakonzepte in Psychotherapie, Beratung, Supervision und Traumapädagogik, Weinheim/Basel: Beltz Juventa 2015.

Gahleitner, Silke Brigitta: Das therapeutische Milieu in der Arbeit mit Kindern und Jugendlichen. Trauma und Beziehungsarbeit in stationären Einrichtungen, Bonn: Psychiatrie-Verlag 2012.

Gahleitner, Silke Brigitta: Traumatisierten Kindern begegnen. Grundlagen der Traumapädagogik, Berlin: ASH 2014.

Die Grundhaltung der BAG Traumapädagogik, in: Lang, Birgit/Schirmer, Claudia/ Lang, Thomas/Andreae de Hair, Ingeborg/Wahle, Thomas/Bausum, Jakob/ Weiß, Wilma/Schmid, Marc: Traumapädagogische Standards in der stationären Kinder- und Jugendhilfe. Eine Praxis- und Orientierungshilfe der BAG Traumapädagogik, Weinheim/Basel: Beltz Juventa 2013, 106-126.

Hermann, Judith: Die Narben der Gewalt. Traumatische Erfahrungen verstehen und überwinden, Paderborn: Junfermann ${ }^{3} 2010$.

Krüger, Andreas: Powerbook - Erste Hilfe für die Seele. Trauma-Selbsthilfe für junge Menschen, Hamburg: Elbe \& Krüger ${ }^{4} 2015$.

Landolt, Markus A.: Psychotraumatologie des Kindesalters, Göttingen: Hogrefe 2004.

Lang, Birgit: Die PädagogInnen als Teil der Pädagogik, in: Lang, Birgit/Schirmer, Claudia/Lang, Thomas/Andreae de Hair, Ingeborg/Wahle, Thomas/Bausum, Jakob/Weiß, Wilma/Schmid, Marc: Traumapädagogische Standards in der stationären Kinder- und Jugendhilfe. Eine Praxis- und Orientierungshilfe der BAG Traumapädagogik, Weinheim/Basel: Beltz Juventa 2013, 127-144.

Lang, Birgit/Wiesinger, Detlev/Schmid, Marc: Das traumapädagogische Konzept der Wohngruppe „Greccio“ in der Umsetzung. Die milieutherapeutische Praxis, in: Trauma und Gewalt (Themenheft Traumapädagogik II) 3/2 (2009) 106-116. 
Lang, Thomas: Bindungspädagogik - Haltgebende, verlässliche und einschätzbare Beziehungsangebote für Kinder und Jugendliche, in: Lang, Birgit/Schirmer, Claudia/Lang, Thomas/Andreae de Hair, Ingeborg/Wahle, Thomas/Bausum, Jakob/Weiß, Wilma/Schmid, Marc: Traumapädagogische Standards in der stationären Kinder- und Jugendhilfe. Eine Praxis- und Orientierungshilfe der BAG Traumapädagogik, Weinheim/Basel: Beltz Juventa 2013, 187-217.

Levine, Peter A./Kline, Maggie: Verwundete Kinderseelen heilen. Wie Kinder und Jugendliche traumatische Erlebnisse überwinden können, München: Kösel 2011.

Schirmer, Claudia: Institutionelle Standards - Worauf es bei traumapädagogischen Konzepten in den Institutionen ankommt, in: Lang, Birgit/Schirmer, Claudia/ Lang, Thomas/Andreae de Hair, Ingeborg/Wahle, Thomas/Bausum, Jakob/ Weiß, Wilma/Schmid, Marc: Traumapädagogische Standards in der stationären Kinder- und Jugendhilfe. Eine Praxis- und Orientierungshilfe der BAG Traumapädagogik, Weinheim/Basel: Beltz Juventa 2013, 241-267.

Terr, Lenore C.: Childhood traumatas. An outline and overview, in: American Journal of Psychiatrie 148 (1991) 10-20.

Weiß, Wilma: Philipp sucht sein Ich. Zum pädagogischen Umgang mit Traumata in den Erziehungshilfen, Weinheim/München: Juventa ${ }^{4} 2008$.

Weiß, Wilma: Selbstbemächtigung/Selbstwirksamkeit - ein traumapädagogischer Beitrag zur Traumaheilung, in: Lang, Birgit/Schirmer, Claudia/Lang, Thomas/ Andreae de Hair, Ingeborg/Wahle, Thomas/Bausum, Jakob/Weiß, Wilma/ Schmid, Marc: Traumapädagogische Standards in der stationären Kinder- und Jugendhilfe. Eine Praxis- und Orientierungshilfe der BAG Traumapädagogik, Weinheim/Basel: Beltz Juventa 2013, 145-156. 